\title{
BMJ Open Association between serum uric acid and atrial fibrillation: a cross-sectional community-based study in China
}

\author{
Yue Chen, ${ }^{1}$ Yunlong Xia, ${ }^{1}$ Xu Han, ${ }^{1}$ Yiheng Yang, ${ }^{1}$ Xiaomeng Yin, ${ }^{1}$ Jing Qiu, ${ }^{2}$ \\ Henghui Liu, ${ }^{3}$ Yong Zhou, ${ }^{4}$ Ying Liu ${ }^{1}$
}

To cite: Chen Y, Xia Y, Han $\mathrm{X}$, et al. Association between serum uric acid and atrial fibrillation: a crosssectional community-based study in China. BMJ Open 2017;7:e019037. doi:10.1136/ bmjopen-2017-019037

- Prepublication history for this paper is available online. To view these files, please visit the journal online(http://dx.doi org/10.1136/bmjopen-2017019037).

Received 10 August 2017 Revised 18 November 2017 Accepted 23 November 2017

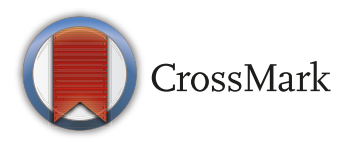

${ }^{1}$ Department of Cardiology, First Affiliated Hospital of Dalian Medical University, Dalian, China ${ }^{2}$ Department of Human Resource, Wuhan Hospital of Traditional Chinese Medicine, Wuhan, China

${ }^{3}$ Department of Technology, Ruike-Donghua Translational Medicine Center Co., Ltd,

Beijing, China

${ }^{4}$ Department of Neurology, Beijing Tiantan Hospital, Capital Medical University, Beijing, China

Correspondence to

Dr Ying Liu;

yingliu.med@gmail.com

\section{ABSTRACT}

\section{Objectives}

To investigate the effects of gender on the association of serum uric acid (SUA) levels and atrial fibrillation (AF) prevalence in a community-based Chinese population. Setting Data were obtained from annual Jidong Oilfield employee and family member health checkups. The Jidong community is geographically located in Tangshan City in northern China.

Participants A total of 9078 residents were invited to take part in the survey and provided informed consents. Individuals without data of ECG or SUA were excluded, leaving 8937 residents in our study.

Primary and secondary outcome measures SUA was measured at baseline using the uricase-peroxidase method. Hyperuricaemia was defined as a SUA level $>7.0 \mathrm{mg} / \mathrm{dL}$ in men and $>5.7 \mathrm{mg} / \mathrm{dL}$ in women. AF was diagnosed based on ECG findings and/or any medical history of AF from referring physicians. The crude and independent association between SUA levels and AF prevalence was evaluated with logistic regression analysis. Results AF prevalence was $0.6 \%$ (53/8937). Participants with hyperuricaemia had a higher AF prevalence compared with those with normal SUA levels (1.1\% vs $0.5 \%$; $\mathrm{P}=0.02$ ). Hyperuricaemia was correlated with $\mathrm{AF}$ after adjustment for various cardiovascular risk factors in all participants ( $P=0.03$, OR $2.051,95 \% \mathrm{Cl} 1.063$ to 3.856$)$. This correlation was particularly stronger in women compared with men $(\mathrm{P}<0.001, \mathrm{OR} 6.366,95 \% \mathrm{Cl} 2.553$ to 15.871 in women and $\mathrm{P}=0.96, \mathrm{OR} 1.025,95 \% \mathrm{Cl} 0.400$ to 2.626 in men).

Conclusions We demonstrated a significant association between increased SUA levels and AF prevalence in a Chinese population. Our data indicate that there is a gender-specific mechanism underlying the relationship between SUA and AF.

\section{INTRODUCTION}

Atrial fibrillation (AF) has become a health concern worldwide because of its significant association with increased risk of morbidity and mortality. The estimated prevalence of $\mathrm{AF}$ is between $2.3 \%$ and $3.4 \%$ in the general population and increases with ageing. ${ }^{12}$ $\mathrm{AF}$ incidence and prevalence are expected to rise continuously over the next few

\section{Strengths and limitations of this study}

The subject group was based on a communitybased population in China, and the sample size of the study was relatively large.

- Our data indicate that there is a gender-specific mechanism underlying the relationship between serum uric acid and atrial fibrillation (AF).

- This was a cross-sectional analysis with a low AF prevalence due to the low mean age of the participants.

- Some AF cases may not have been included in our analyses because participants with paroxysmal AF or asymptomatic AF 'escaped' ECG detection. Therefore, we may have underestimated the AF prevalence.

decades. ${ }^{3}$ A number of factors, including advancing age, male gender, obesity, hypertension, congestive heart failure, myocardial infarction (MI), chronic kidney disease and diabetes mellitus, have been identified as risk factors for AF development. ${ }^{4-6}$ Mechanistically, AF occurrence and perpetuation depend on atrial electrophysiological and structural remodelling. ${ }^{7}$ Although the pathophysiology of atrial remodelling is not well understood, recent studies have suggested that inflammation and oxidative stress have been shown to play a role in atrial electrical and/or structural remodelling. ${ }^{8-11}$

Serum uric acid (SUA) has been reported to be clearly associated with oxidative stress and inflammation in several pathological conditions. ${ }^{12}{ }^{13}$ Previous studies explored the role of SUA in AF aetiology and persistence. ${ }^{14} 15$ For instance, several cross-sectional studies, which enrolled hospital patients, showed that elevated SUA levels were linked to a high risk of $\mathrm{AF}^{16}{ }^{17}$ Another study investigated the relationship between SUA and AF based on a community cohort. ${ }^{18}$ In addition, a large meta-analysis confirmed a potential association between SUA and AF. ${ }^{19}$ Although some studies analysed the effect of gender on the 
relationship between elevated SUA levels and AF and showed a gender-specific mechanism, ${ }^{17}{ }^{18}$ the findings were not consistent. Therefore, the aim of the present study was to further investigate the relationship between SUA levels and AF prevalence in a relatively large, community-based cohort in China and analyse the independent effects of gender on the association between SUA and AF.

\section{METHODS}

\section{Study design and population}

The current study included participants in the population-based Cohort Study in Outcome of Phased Progression of Atherosclerosis. Between April and August of 2014, data were obtained from annual Jidong Oilfield employee and family member health checkups. The Jidong community is geographically located in Tangshan City in northern China. All residents aged 18 years or older in the Jidong community were invited to participate in our study. A total of 9078 residents finally agreed to take part in the survey and provided informed consents; all of the 9078 residents underwent health examinations and filled in the questionnaires. Individuals without data of ECG or SUA were excluded, leaving 8937 residents in our study.

\section{Definition of atrial fibrillation}

In our study, all participants were subjected to a 12-lead ECG at rest for $5 \mathrm{~min}$. All participants avoided drinking alcohol and tea before examination. Recordings were then transferred to a computer for data interpretation. Each ECG rhythm strip was read back-to-back by two research doctors from the Medical Centers of the Jidong Oilfield. If outcomes differed, ECGs were further reviewed by an experienced cardiologist. If anyone self-reported a medical history of AF, but the ECG was negative, we confirmed the case with referring physicians and obtained the data to confirm the diagnosis. AF was diagnosed if one of the following criteria was present: (1) irregular R-R intervals, (2) absence of repeating $\mathrm{P}$ waves and (3) irregular atrial activity.

\section{Definition of hyperuricaemia}

Baseline SUA measurements were reported in $\mu \mathrm{mol} / \mathrm{L}$, and we converted those values using the following conversion equivalence: $1 \mathrm{mg} / \mathrm{dL}=59.48 \mu \mathrm{mol} / \mathrm{L}$. SUA was determined using the uricase-peroxidase method. Hyperuricaemia was defined as an SUA concentration $>7.0 \mathrm{mg} /$ $\mathrm{dL}$ in men and $>5.7 \mathrm{mg} / \mathrm{dL}$ in women or current use of uric acid (UA)-lowering drugs. ${ }^{20}$

\section{Biochemical measurements}

Blood samples were drawn from the antecubital vein of each participant in the morning in the fasting state and stored in vacuum tubes containing EDTA. We measured serum creatinine, UA, total cholesterol (TC), triglycerides (TG), high-density lipoprotein-cholesterol (HDL-c), low-density lipoprotein-cholesterol (LDL-c) and fasting blood glucose
(FBG) in all participants. The estimated glomerular filtration rate (eGFR) was calculated using a formula based on the Chronic Kidney Disease Epidemiology Collaboration equation $^{21}$ as follows: GFR $=141 \times \min (\mathrm{Scr} / \kappa, 1)^{\alpha} \times \max -$ $(\mathrm{Scr} / \kappa, 1)^{-1.209} \times 0.993^{\mathrm{Age}} \times 1.018$ (if female, or) $\times 1.159$ (if black), where $\kappa$ is 0.7 for female and 0.9 for male; $\alpha$ is 0.329 for female and 0.411 for male; min indicates the minimum of SC/ $/$ or 1 ; and max indicates the maximum of $\mathrm{SC} / \kappa$.

\section{Definition of clinical variables}

Demographic characteristics (age, sex, weight, body height, alcohol and cigarette use) and clinic characteristics (history of hypertension, diabetes mellitus, heart failure, gout, dyslipidaemia, MI, transient ischaemic attack (TIA), AF and medicine use) were obtained from physical examinations and questionnaires by research doctors from Medical Centers of Jidong Oilfield, as described in previous studies. ${ }^{22}{ }^{23}$ We measured body weight (to the nearest $0.1 \mathrm{~kg}$ ) and body height (to the nearest $0.1 \mathrm{~cm}$ ) with participants wearing light clothing and no shoes, and body mass index (BMI) was calculated as body weight $(\mathrm{kg})$ divided by height $\left(\mathrm{m}^{2}\right)$. Hypertension was defined as a history of arterial hypertension or blood pressure $\geq 140 / 90 \mathrm{~mm} \mathrm{Hg}$ (mean of three measurements) in the supine position or current treatment with any antihypertensive medications. Diabetes mellitus was defined by a self-reported history, the use of insulin, oral hypoglycaemic agents or FBG $\geq 7 \mathrm{mmol} / \mathrm{L}$. Dyslipidaemia was defined by a self-reported history, current use of cholesterol-lowering medicine, TC level $\geq 5.7 \mathrm{mmol} / \mathrm{L}$, $\mathrm{TG} \geq 1.7 \mathrm{mmol} / \mathrm{L}$ or $\mathrm{LDL} \geq 4.1 \mathrm{mmol} / \mathrm{L}$.

\section{Statistical analysis}

All statistical analyses were performed with the SAS software V.9.4. The distributions (mean $\pm \mathrm{SD}$ ) of the parameters were calculated. Continuous variables (with or without hyperuricaemia) were performed using the analysis of variance analysis, while the $\chi^{2}$ test was applied for comparing categorical variables. We performed collinearity analysis of variables prior to logistic regression analysis, which showed that eGFR and BMI are collinear variables, and these two indexes were therefore excluded from the adjusted model. Univariate and multivariate logistic regression analysis was used to analyse the associations between SUA and AF prevalence. The OR was presented with their corresponding $95 \%$ CIs. A $\mathrm{P}$ value of $<0.05$ (two-tailed) was considered statistically significant.

\section{RESULTS}

\section{Subject characteristics}

Data were obtained from 8937 participants aged 18-82 years, including $4686(52.4 \%)$ men and $4251(47.6 \%)$ women, and the mean age was $42.1 \pm 13.1$ years. Participants were divided into two groups on the basis of SUA levels: normal SUA-level group and hyperuricaemia 
Table 1 Comparison of demographic and clinical characteristics of participants between normal and high SUA-level groups

\begin{tabular}{|c|c|c|c|c|}
\hline Subjects & Total $(n=8937)$ & Normal SUA level $(n=7740)$ & Hyperuricaemia $(n=1197)$ & $\mathbf{P}$ value \\
\hline Age, years & $42.1 \pm 13.1$ & $42.2 \pm 13.0$ & $41.6 \pm 13.6$ & 0.10 \\
\hline Male, N (\%) & $4686(52.4 \%)$ & $3844(49.7 \%)$ & $842(70.3 \%)$ & $<0.001$ \\
\hline BMI $\left(\mathrm{kg} / \mathrm{m}^{2}\right)$ & $24.5 \pm 3.7$ & $24.2 \pm 3.6$ & $26.8 \pm 3.7$ & $<0.001$ \\
\hline Smoking, N (\%) & $2324(26.0 \%)$ & $1912(24.7 \%)$ & $412(34.4 \%)$ & $<0.001$ \\
\hline Alcohol use, N (\%) & $2971(33.2 \%)$ & $2421(31.3 \%)$ & $550(45.9 \%)$ & $<0.001$ \\
\hline Hypertension, N (\%) & 2777 (31.1\%) & $2215(28.6 \%)$ & $562(47.0 \%)$ & $<0.001$ \\
\hline Diabetes, N (\%) & $596(6.7 \%)$ & $512(6.6 \%)$ & $84(7.0 \%)$ & 0.60 \\
\hline Heart failure, N (\%) & $7(0.08 \%)$ & $5(0.06 \%)$ & $2(0.17 \%)$ & 0.24 \\
\hline Gout, N (\%) & $35(0.39 \%)$ & $17(0.22 \%)$ & $18(1.50 \%)$ & $<0.001$ \\
\hline Dyslipidaemia, N (\%) & 3256 (36.4\%) & $2540(32.8 \%)$ & $716(59.8 \%)$ & $<0.001$ \\
\hline MI, N (\%) & $41(0.5 \%)$ & $37(0.5 \%)$ & $4(0.3 \%)$ & 0.49 \\
\hline TIA/stroke, N (\%) & $82(0.9 \%)$ & $72(0.9 \%)$ & $10(0.8 \%)$ & 0.75 \\
\hline Statins use, N (\%) & $110(1.2 \%)$ & $87(1.1 \%)$ & $23(1.9 \%)$ & 0.02 \\
\hline Diuretic use, N (\%) & $51(0.6 \%)$ & $34(0.4 \%)$ & $17(1.4 \%)$ & $<0.001$ \\
\hline $\mathrm{TC}(\mathrm{mmol} / \mathrm{L})$ & $4.4 \pm 0.9$ & $4.4 \pm 0.9$ & $4.7 \pm 0.9$ & $<0.001$ \\
\hline $\mathrm{TG}(\mathrm{mmol} / \mathrm{L})$ & $1.6 \pm 1.4$ & $1.5 \pm 1.2$ & $2.3 \pm 2.0$ & $<0.001$ \\
\hline HDL-c (mmol/L) & $1.2 \pm 0.3$ & $1.2 \pm 0.3$ & $1.1 \pm 0.2$ & $<0.001$ \\
\hline LDL-c (mmol/L) & $2.5 \pm 0.6$ & $2.4 \pm 0.6$ & $2.7 \pm 0.6$ & $<0.001$ \\
\hline FBG (mmol/L) & $5.3 \pm 1.1$ & $5.2 \pm 1.1$ & $5.4 \pm 1.1$ & $<0.001$ \\
\hline Creatinine $(\mu \mathrm{mol} / \mathrm{L})$ & $77.2 \pm 16.1$ & $76.1 \pm 14.8$ & $84.4 \pm 21.8$ & $<0.001$ \\
\hline eGFR (median) (mL/min/1.73 $\left.\mathrm{m}^{2}\right)$ & $97.6 \pm 16.3$ & $98.1 \pm 15.8$ & $94.1 \pm 18.3$ & $<0.001$ \\
\hline $\mathrm{AF}, \mathrm{N}(\%)$ & $53(0.6 \%)$ & $40(0.5 \%)$ & $13(1.1 \%)$ & 0.02 \\
\hline SUA (mg/dL) & $5.0 \pm 1.5$ & $4.6 \pm 1.2$ & $7.5 \pm 1.1$ & $<0.001$ \\
\hline
\end{tabular}

Values are expressed as mean \pm SD or numbers (percentage).

AF, atrial fibrillation; BMI, body mass index; eGFR, estimated glomerular filtration rate; FBG, fasting blood glucose; HDL-c, high-density lipoprotein-cholesterol; LDL-c, low-density lipoprotein-cholesterol; MI, myocardial infarction; SUA, serum uric acid; TG, triglycerides; TC, total cholesterol; TIA, transient ischaemic attack .

group. The average SUA level was $5.0 \pm 1.5 \mathrm{mg} / \mathrm{dL}$, and the prevalence of hyperuricaemia was $13.4 \%$. Baseline characteristics, medical histories and biochemical markers of participants in the presence or absence of hyperuricaemia are presented in table 1. Male, hypertension, dyslipidaemia, gout, smoking, alcohol use, statin use and diuretic use were more prevalent in the subjects with hyperuricaemia (all $\mathrm{P}<0.05$ ). Compared with patients with normal SUA levels, those with hyperuricaemia had a significantly higher BMI, TC, TG, LDL-c and FBG levels $($ all $\mathrm{P}<0.05)$. However, there were no significant differences between these two groups in terms of age, diabetes, heart failure, MI and TIA/stroke. We also summarised the clinical characteristics between the AF group and non-AF group in table 2.

\section{Association of SUA level with AF}

AF prevalence was $0.6 \%(53 / 8937)$ in all subjects, $0.7 \%$ $(35 / 4686)$ in men and $0.4 \%(18 / 4251)$ in women. The correlation between hyperuricaemia and AF was assessed by logistic regression analysis for non-adjusted and adjusted models (table 3). Patients in the hyperuricaemia group had a higher $\mathrm{AF}$ prevalence compared with those in the normal SUA group in all subjects $(\mathrm{P}=0.02$, OR $2.114,95 \%$ CI 1.128 to 3.964$)$ and in women $(\mathrm{P}<0.001$, OR $7.104,95 \% \mathrm{CI} 2.737$ to 18.442$)$, but not in men $(\mathrm{P}=0.90$, OR $0.944,95 \%$ CI 0.391 to 2.281). The association with AF remained significant in all subjects following simultaneous adjustment for age, hypertension, diabetes mellitus, dyslipidaemia, smoking, alcohol use, statins use, diuretic use, history of MI, TIA/stroke, heart failure and gout $(\mathrm{P}=0.03$, OR 2.051, 95\% CI 1.063 to $3.856)$ and in women $(\mathrm{P}<0.001$, OR $6.366,95 \%$ CI 2.553 to 15.871). Additionally, we conducted a sensitivity analysis to evaluate the robustness of our findings. An almost identical result was found when we excluded participants with hypertension ( $\mathrm{P}<0.001$, OR 5.666, 95\% CI 2.383 to 13.473). Similar correlations were observed when we excluded subjects with $\mathrm{MI}$ or heart failure ( $\mathrm{P}=0.02$, OR $2.183,95 \%$ CI 1.132 to 4.212 ).

\section{DISCUSSION}

Several previous studies suggested that elevated SUA levels were positively associated with AF prevalence in 
Table 2 Comparison of demographic and clinical characteristics of participants between AF and non-AF group

\begin{tabular}{|c|c|c|c|c|}
\hline Subjects & Total $(n=8937)$ & Non-AF group $(n=8884)$ & AF group $(n=53)$ & $P$ value \\
\hline Age (years) & $42.1 \pm 13.1$ & $42.1 \pm 13.1$ & $56.1 \pm 11.6$ & $<0.001$ \\
\hline Male, N (\%) & $4686(52.4 \%)$ & $4651(52.4 \%)$ & $35(66.0 \%)$ & 0.04 \\
\hline $\mathrm{BMI}\left(\mathrm{kg} / \mathrm{m}^{2}\right)$ & $24.5 \pm 3.7$ & $24.5 \pm 3.7$ & $25.2 \pm 2.8$ & 0.08 \\
\hline Smoking, N (\%) & $2324(26.0 \%)$ & $2308(26.0 \%)$ & $16(30.2 \%)$ & 0.49 \\
\hline Alcohol use, N (\%) & $2971(33.2 \%)$ & $2951(33.2 \%)$ & $20(37.7 \%)$ & 0.49 \\
\hline Hypertension, N (\%) & 2777 (31.1\%) & $2750(31.0 \%)$ & 27 (50.9\%) & 0.01 \\
\hline Diabetes, N (\%) & $596(6.7 \%)$ & $586(6.6 \%)$ & $10(18.9 \%)$ & $<0.001$ \\
\hline Heart failure, $\mathrm{N}(\%)$ & $7(0.08 \%)$ & $6(0.1 \%)$ & $1(1.9 \%)$ & $<0.001$ \\
\hline Gout, N (\%) & $35(0.39 \%)$ & $34(0.4 \%)$ & $1(1.9 \%)$ & 0.08 \\
\hline Dyslipidaemia, N (\%) & $3256(36.4 \%)$ & $3228(36.3 \%)$ & $28(52.8 \%)$ & 0.01 \\
\hline MI, N (\%) & $41(0.5 \%)$ & $38(0.4 \%)$ & $3(5.7 \%)$ & $<0.001$ \\
\hline TIA/stroke, N (\%) & $82(0.9 \%)$ & $80(0.9 \%)$ & $2(3.8 \%)$ & 0.03 \\
\hline Statins use, N (\%) & $110(1.2 \%)$ & $103(1.2 \%)$ & $7(13.2 \%)$ & $<0.001$ \\
\hline Diuretic use, N (\%) & $51(0.6 \%)$ & $50(0.6 \%)$ & $1(1.9 \%)$ & 0.20 \\
\hline $\mathrm{TC}(\mathrm{mmol} / \mathrm{L})$ & $4.4 \pm 0.9$ & $4.4 \pm 0.9$ & $4.4 \pm 0.9$ & 0.99 \\
\hline $\mathrm{TG}(\mathrm{mmol} / \mathrm{L})$ & $1.6 \pm 1.4$ & $1.6 \pm 1.3$ & $2.0 \pm 1.1$ & 0.37 \\
\hline $\mathrm{HDL}-\mathrm{c}(\mathrm{mmol} / \mathrm{L})$ & $1.2 \pm 0.3$ & $1.2 \pm 0.3$ & $1.1 \pm 0.3$ & 0.13 \\
\hline LDL-c (mmol/L) & $2.5 \pm 0.6$ & $2.5 \pm 0.6$ & $2.5 \pm 0.5$ & 0.82 \\
\hline FBG $(\mathrm{mmol} / \mathrm{L})$ & $5.3 \pm 1.1$ & $5.3 \pm 1.1$ & $5.8 \pm 1.3$ & $<0.001$ \\
\hline Creatinine $(\mu \mathrm{mol} / \mathrm{L})$ & $77.2 \pm 16.1$ & $77.1 \pm 16.1$ & $80.1 \pm 12.3$ & 0.09 \\
\hline $\begin{array}{l}\text { eGFR (median) (mL/ } \\
\left.\min / 1.73 \mathrm{~m}^{2}\right)\end{array}$ & $97.6 \pm 16.3$ & $97.6 \pm 16.2$ & $87.8 \pm 15.8$ & $<0.001$ \\
\hline Hyperuricaemia, N (\%) & $1197(13.4 \%)$ & $1184(13.3 \%)$ & $13(24.5 \%)$ & 0.02 \\
\hline SUA (mg/dL) & $5.0 \pm 1.5$ & $5.0 \pm 1.5$ & $5.5 \pm 1.4$ & 0.01 \\
\hline
\end{tabular}

Values are expressed as mean \pm SD or numbers (percentage).

$\mathrm{AF}$, atrial fibrillation; BMI, body mass index; eGFR, estimated glomerular filtration rate; FBG, fasting blood glucose; HDL-c, high-density lipoprotein-cholesterol; LDL-c, low-density lipoprotein-cholesterol; MI, myocardial infarction; SUA, serum uric acid; TC, total cholesterol; TG, triglycerides; TIA, transient ischaemic attack .

both hypertensive ${ }^{24}$ and chronic systolic heart failure patients. ${ }^{25}$ A recent meta-analysis, which included both cross-sectional and cohort studies, also demonstrated a positive relationship between SUA and $\mathrm{AF}^{26}$ In the present study, we explored the relationship between SUA levels and $\mathrm{AF}$ in a Chinese community-based population and found that elevated SUA levels were significantly correlated with AF, and that the independent association between these two persisted after adjusting for multiple clinical factors in the general population, especially in women. In addition, the AF prevalence was relatively low on account of the low mean age (42 years), which contributed to AF's association with other risk factors. The logistic regression analysis was helpful for adjusting this bias and increasing the reliability of the findings. In the present study, we found that the width and magnitude of the observed CIs of total subjects and men were acceptable; however, the CIs of women were relatively wide. The reasons were probably linked to the low $\mathrm{AF}$ prevalence and the difference of AF prevalence in different genders with hyperuricaemia, as evidenced by the finding that $\mathrm{AF}$ prevalence was higher in women with hyperuricaemia. In addition, although hyperuricaemia was more prevalent in men than women $(18 \%$ vs $8.4 \%, \mathrm{P}<0.001)$, hyperuricaemia was associated with AF in women but not in men, partly because being a woman affects the relationship between SUA and AF.

Consistent with a previous report, ${ }^{15}$ we show here that elevated SUA levels are positively correlated with an increased risk of $\mathrm{AF}$ in a general Chinese population. Moreover, we found that SUA has gender-specific, independent effects on AF prevalence in women, but not in men, which was in accordance with a previous study in Japan, ${ }^{17}$ which was based on a relatively large hospital-based cohort with a high prevalence of $\mathrm{AF}$ and divided patients according to sex-specific UA tertiles. This study showed that gender affected the independent relationship between SUA and AF after adjusting for well-known cardiovascular risk factors. Our findings were also consistent with several previous studies that reported gender-specific effects of SUA on the prevalence of hypertension and kidney disease in women. ${ }^{27} 28$ 
Table 3 OR and $95 \% \mathrm{Cl}$ between hyperuricaemia and $\mathrm{AF}$ prevalence

\begin{tabular}{|c|c|c|c|c|c|c|c|}
\hline \multirow[b]{2}{*}{ Group } & \multirow[b]{2}{*}{ Subjects (n) } & \multirow[b]{2}{*}{ AF } & \multirow[b]{2}{*}{ Prevalence (\%) } & \multicolumn{2}{|l|}{ Unadjusted model } & \multicolumn{2}{|l|}{ Adjusted model } \\
\hline & & & & OR $(95 \% \mathrm{Cl})$ & $P$ value & OR $(95 \% \mathrm{Cl})$ & $\mathbf{P}$ value \\
\hline Men* & 4686 & 35 & 0.74 & & & & \\
\hline Hyperuricaemia & 842 & 6 & 0.71 & 0.944 (0.391 to 2.281$)$ & 0.90 & 1.025 (0.400 to 2.626$)$ & 0.96 \\
\hline Women* & 4251 & 18 & 0.42 & & & & \\
\hline Hyperuricaemia & 355 & 7 & 1.97 & 7.104 (2.737 to 18.442$)$ & $<0.001$ & $6.366(2.553$ to 15.871$)$ & $<0.001$ \\
\hline Total $†$ & 8937 & 53 & 0.60 & & & & \\
\hline Normal SUA level & 7740 & 40 & 0.52 & 1.000 & 1.0000 & 1.000 & 1.0000 \\
\hline Hyperuricaemia & 1197 & 13 & 1.09 & 2.114 (1.128 to 3.964$)$ & 0.02 & 2.051 (1.063 to 3.856$)$ & 0.03 \\
\hline \multicolumn{8}{|l|}{ Sensitive analysis } \\
\hline Hyperuricaemia & 635 & 9 & 1.42 & 4.658 (2.068 to 10.494$)$ & $<0.001$ & $5.666(2.383$ to 13.473$)$ & $<0.001$ \\
\hline \multicolumn{8}{|l|}{ Total $\S$} \\
\hline Normal SUA level & 7699 & 37 & 0.48 & 1.000 & 1.0000 & 1.000 & 1.0000 \\
\hline Hyperuricaemia & 1191 & 13 & 1.09 & 2.285 (1.211 to 4.312$)$ & 0.01 & 2.183 (1.132 to 4.212$)$ & 0.02 \\
\hline
\end{tabular}

*Model 1: adjusted for age, smoking, alcohol use, diuretics use, statins use, hypertension, diabetes, MI, TIA/stroke and dyslipidaemia, heart failure and gout.

†Model 2: adjusted for model 1 and sex

$¥$ Model 3: adjusted for model 2 and further excluded subjects with hypertension.

§Model 4: adjusted for model 2 and further excluded subjects with MI or heart failure.

AF, atrial fibrillation; MI, myocardial infarction; SUA, serum uric acid; TIA, transient ischaemic attack.

Possible mechanism of gender difference is potentially linked, in part, to menopause, as oestrogen is known to be uricosuric. In our study, nearly one-third of women (26.11\%) were postmenopausal, thus, we believe that age-related postmenopause potentially contributed to the possible mechanism underlying the gender difference. A recent study proposed that independent association with SUA was only observed in the total population and in men after adjusting for other cardiovascular risk factors. However, this study only enrolled patients aged $\geq 35$ years in the rural Liaoning province of China. ${ }^{18}$ Thus, it is possible that the differences in age, region and dietary customs account for the observed discrepancies between the present and previous studies. Further studies with a larger sample size are needed to confirm the regional and gender-specific link between SUA and AF.

As noted above, some epidemiological studies suggested an association between elevated SUA levels and $\mathrm{AF}$, but little is known about the pathophysiological mechanism underlying this link. However, SUA biology may point to a potential mechanism. SUA is the final product of purine metabolism catalysed by xanthine oxidase, which plays an important role in the formation of free radical superoxide anion and oxidative stress, consequently resulting in calcium overload and decreasing sodium channels and aggravating cellular damage. These pathological processes promote electrical remodelling of the left atria. ${ }^{29}{ }^{30}$ In addition,
SUA promotes inflammation via releasing pro-inflammatory cytokines (eg, interleukin-6, interleukin-8, tumour necrosis factor and monocyte chemoattractant protein 1$)^{31-33}$ or local activation of the renin-angiotensin system (RAS) ${ }^{34}$ It is well known that chronic inflammation leads to endothelial activation/damage, production of tissue factor from monocytes, increased platelet activation and elevated expression of fibrinogen. ${ }^{35}$ Inflammation also upregulates the RAS and increases angiotensin II (Ang II) levels, which activate the Janus kinase (JAK)/signal transducers and activators of transcription (STAT) pathway. It has been shown that the Ang II/Rac1/STAT3 pathway is an important signalling pathway that can promote $\mathrm{AF}^{37}$ Both JAK/ STAT and RAS signalling play a role in left atrial structural remodelling, and both electrical and structural remodelling contribute to the occurrence and development of AF. ${ }^{38}$ Another potential epidemiological explanation for the relationship between SUA and AF could be that SUA is an independent predictor of various cardiovascular diseases, including stroke, hypertension, coronary heart disease and congestive heart failure. ${ }^{39-41}$ Thus, elevated SUA levels may lead to increased AF prevalence via increasing the risk of cardiovascular diseases.

Our study has several limitations. First, this was a cross-sectional analysis with a low AF prevalence due to the low mean age of the participants. Furthermore, we did not explore the cause and effect of elevated SUA levels 
and $\mathrm{AF}$ because this association may have been affected by reverse causality and survivor bias in the cross-sectional study. Second, the data were obtained from a population in northern China, and the lifestyle and diet customs (eg, meat, seafood and milk), which are different from other regions of China, played a significant role in SUA metabolism. ${ }^{42}$ Therefore, this study was not nationally representative or regionally diverse. Third, some AF cases may not have been included in our analyses because participants with paroxysmal AF or asymptomatic AF 'escaped' ECG detection, and some subjects with valvular AF might not be excluded. Therefore, AF prevalence may be biased. Despite these limitations, our study has some important strengths. Specifically, the subject group was based on community-based population in China, and the sample size of the study was relatively large.

\section{CONCLUSION}

In conclusion, SUA levels were significantly associated with $\mathrm{AF}$ prevalence in a particular Chinese population. Also, women, but not men, with higher SUA levels tended to have an increased AF prevalence after adjusting for multiple clinical AF risk factors, suggesting a gender-specific mechanism. Further research should focus on confirming our finding and elucidating the exact pathophysiological and prognostic mechanisms underlying the link between SUA and AF.

Acknowledgements The authors appreciate all the participants, their families and the members of the study team from the Jidong community. They thank the staff at Recovery Medical Technology Development Co., Ltd. for their important efforts.

Contributors $Y C$ and $Y X$ contributed equally to this work. $Y C, Y Y$ and $Y X$ provided the conception and design of the study. YZ provided the database. XH, HL and JQ conducted the analysis and interpretation of data. $Y C$ and $Y X$ drafted this paper. $X Y$, $Y L$ and $Y Y$ revised the paper critically for important intellectual content. $Y Z$ approved of the version published. YL agreed to be accountable for all aspects of the work in ensuring that questions related to the accuracy or integrity of any part of work are appropriately investigated and resolved.

Funding The authors thank the support of research grants from the National 12th Five-Year Major Projects of China (2012BAI37B03), the Recovery Medical Science Foundation and the National Natural Science Foundation of China (81570313), and Liaoning Health and Family Planning Commission of China (LNCCC-B04-2014).

Competing interests None declared.

Patient consent Obtained.

Ethics approval The study was conducted according to the guidelines of the Helsinki Declaration and was approved by the Ethics Committee of Jidong Oilfield Medical Centers.

Provenance and peer review Not commissioned; externally peer reviewed.

Data sharing statement The data sets generated and analysed during the current study are available from the corresponding author upon reasonable request.

Open Access This is an Open Access article distributed in accordance with the Creative Commons Attribution Non Commercial (CC BY-NC 4.0) license, which permits others to distribute, remix, adapt, build upon this work non-commercially, and license their derivative works on different terms, provided the original work is properly cited and the use is non-commercial. See: http://creativecommons.org/ licenses/by-nc/4.0/

(C) Article author(s) (or their employer(s) unless otherwise stated in the text of the article) 2017. All rights reserved. No commercial use is permitted unless otherwise expressly granted.
REFERENCES

1. Ball J, Carrington MJ, McMurray JJ, et al. Atrial fibrillation: profile and burden of an evolving epidemic in the 21st century. Int $J$ Cardiol 2013;167:1807-24

2. Lip GYH, Brechin CM, Lane DA. The global burden of atrial fibrillation and stroke: a systematic review of the epidemiology of atrial fibrillation in regions outside North America and Europe. Chest 2012;142:1489-98.

3. Miyasaka Y, Barnes ME, Gersh BJ, et al. Secular trends in incidence of atrial fibrillation in Olmsted County, Minnesota, 1980 to 2000, and implications on the projections for future prevalence. Circulation 2006;114:119-25.

4. Schnabel RB, Sullivan LM, Levy D, et al. Development of a risk score for atrial fibrillation (Framingham Heart Study): a community-based cohort study. Lancet 2009;373:739-45.

5. Alonso A, Lopez FL, Matsushita K, et al. Chronic kidney disease is associated with the incidence of atrial fibrillation: the Atherosclerosis Risk in Communities (ARIC) study. Circulation 2011;123:2946-53.

6. Horio T, Iwashima Y, Kamide K, et al. Chronic kidney disease as an independent risk factor for new-onset atrial fibrillation in hypertensive patients. J Hypertens 2010;28:1738-44.

7. Nattel S, Burstein B, Dobrev D. Atrial remodeling and atrial fibrillation: mechanisms and implications. Circ Arrhythm Electrophysiol 2008;1:62-73.

8. Berry CE, Hare JM. Xanthine oxidoreductase and cardiovascular disease: molecular mechanisms and pathophysiological implications. J Physiol 2004;555(Pt 3):589-606.

9. Ghaemi-Oskouie F, Shi Y. The role of uric acid as an endogenous danger signal in immunity and inflammation. Curr Rheumatol Rep 2011;13:160-6.

10. Shi Y, Evans JE, Rock KL. Molecular identification of a danger signal that alerts the immune system to dying cells. Nature 2003;425:516-21.

11. Patel $P$, Dokainish $H$, Tsai $P$, et al. Update on the association of inflammation and atrial fibrillation. $J$ Cardiovasc Electrophysiol 2010;21:1064-70.

12. Strazzullo P, Puig JG. Uric acid and oxidative stress: relative impact on cardiovascular risk? Nutr Metab Cardiovasc Dis 2007;17:409-14.

13. Kanellis J, Kang DH. Uric acid as a mediator of endothelial dysfunction, inflammation, and vascular disease. Semin Nephrol 2005;25:39-42.

14. Chuang S-Y, Wu C-C, Hsu P-F, et al. Hyperuricemia and incident atrial fibrillation in a normotensive elderly population in Taiwan. Nutrition, Metabolism and Cardiovascular Diseases 2014;24:1020-6.

15. Nyrnes A, Toft I, Njølstad I, et al. Uric acid is associated with future atrial fibrillation: an 11-year follow-up of 6308 men and women--the Tromso Study. Europace 2014;16:320-6.

16. Letsas KP, Korantzopoulos P, Filippatos GS, et al. Uric acid elevation in atrial fibrillation. Hellenic J Cardiol 2010;51:209-13.

17. Suzuki S, Sagara K, Otsuka T, et al. Gender-specific relationship between serum uric acid level and atrial fibrillation prevalence. Circ $J$ 2012;76:607-11.

18. Sun GZ, Guo L, Wang J, et al. Association between hyperuricemia and atrial fibrillation in rural China: a cross-sectional study. BMC Cardiovasc Disord 2015;15:98.

19. Xu X, Du N, Wang R, et al. Hyperuricemia is independently associated with increased risk of atrial fibrillation: A meta-analysis of cohort studies. Int J Cardiol 2015;184:699-702.

20. Zhu Y, Pandya BJ, Choi HK. Prevalence of gout and hyperuricemia in the US general population: the national health and nutrition examination survey 2007-2008. Arthritis Rheum 2011;63:3136-41.

21. Levey AS, Stevens LA, Schmid CH, et al. A new equation to estimate glomerular filtration rate. Ann Intern Med 2009;150:604-12.

22. Li Z, Yang X, Wang A, et al. Association between ideal cardiovascular health metrics and depression in Chinese population: a crosssectional study. Sci Rep 2015;5:11564.

23. Song $Q$, Liu X, Wang A, et al. Associations between non-traditional lipid measures and risk for type 2 diabetes mellitus in a Chinese community population: a cross-sectional study. Lipids Health Dis 2016;15:70.

24. Liu T, Zhang X, Korantzopoulos $P$, et al. Uric acid levels and atrial fibrillation in hypertensive patients. Intern Med 2011;50:799-803.

25. Liu Y, Liu H, Dong L, et al. Prevalence of atrial fibrillation in hospitalized patients over 40 years old: ten-year data from the People's Hospital of Peking University. Acta Cardiol 2010;65:221-4.

26. Tamariz L, Hernandez F, Bush A, et al. Association between serum uric acid and atrial fibrillation: a systematic review and meta-analysis. Heart Rhythm 2014;11:1102-8.

27. Zhang W, Sun K, Yang Y, et al. Plasma uric acid and hypertension in a Chinese community: prospective study and metaanalysis. Clin Chem 2009;55:2026-34. 
28. Weiner DE, Tighiouart H, Elsayed EF, et al. Uric acid and incident kidney disease in the community. J Am Soc Nephrol 2008;19:1204-11.

29. Korantzopoulos P, Kolettis TM, Galaris D, et al. The role of oxidative stress in the pathogenesis and perpetuation of atrial fibrillation. Int $J$ Cardiol 2007;115:135-43.

30. Dudley SC, Hoch NE, McCann LA, et al. Atrial fibrillation increases production of superoxide by the left atrium and left atrial appendage: role of the NADPH and xanthine oxidases. Circulation 2005;112:1266-73.

31. Kang DH, Han L, Ouyang X, et al. Uric acid causes vascular smooth muscle cell proliferation by entering cells via a functional urate transporter. Am J Nephrol 2005;25:425-33.

32. Kanellis J, Watanabe S, Li JH, et al. Uric acid stimulates monocyte chemoattractant protein-1 production in vascular smooth muscle cells via mitogen-activated protein kinase and cyclooxygenase-2. Hypertension 2003;41:1287-93.

33. Baldwin W, McRae S, Marek G, et al. Hyperuricemia as a mediator of the proinflammatory endocrine imbalance in the adipose tissue in a murine model of the metabolic syndrome. Diabetes 2011;60:1258-69.

34. Johnson RJ, Rodriguez-lturbe B, Kang DH, et al. A unifying pathway for essential hypertension. Am J Hypertens 2005;18:431-40.
35. Aviles RJ, Martin DO, Apperson-Hansen C, et al. Inflammation as a risk factor for atrial fibrillation. Circulation 2003;108:3006-10.

36. Guo Y, Lip GY, Apostolakis S. Inflammation in atrial fibrillation. J Am Coll Cardiol 2012;60:2263-70.

37. Tsai CT, Lai LP, Kuo KT, et al. Angiotensin II activates signal transducer and activators of transcription 3 via Rac1 in atrial myocytes and fibroblasts: implication for the therapeutic effect of statin in atrial structural remodeling. Circulation 2008;117:344-55.

38. Korantzopoulos P, Letsas KP, Liu T. Xanthine oxidase and uric Acid in atrial fibrillation. Front Physiol 2012;3:150.

39. Kanbay M, Yilmaz MI, Sonmez A, et al. Serum uric acid independently predicts cardiovascular events in advanced nephropathy. Am J Nephrol 2012;36:324-31.

40. Strasak A, Ruttmann E, Brant L, et al. Serum uric acid and risk of cardiovascular mortality: a prospective long-term study of 83,683 Austrian men. Clin Chem 2008;54:273-84.

41. Feig DI, Kang DH, Johnson RJ. Uric acid and cardiovascular risk N Engl J Med 2008;359:1811-21.

42. Torralba KD, De Jesus E, Rachabattula S. The interplay between diet, urate transporters and the risk for gout and hyperuricemia: current and future directions. Int J Rheum Dis 2012;15:499-506. 